\title{
Erratum: NLO QCD + NLO EW corrections to WZZ productions with leptonic decays at the LHC
}

\author{
Shen Yong-Bai, ${ }^{a}$ Zhang Ren-You, ${ }^{a}$ Ma Wen-Gan, ${ }^{a}$ Li Xiao-Zhou, ${ }^{a}$ Zhang $\mathrm{Yu}^{a}$ and \\ Guo Lei $^{b}$ \\ ${ }^{a}$ Department of Modern Physics, University of Science and Technology of China, \\ 96 Jinzhai Road, Hefei, Anhui, 230026 P.R. China \\ ${ }^{b}$ Department of Physics, Chongqing University, \\ 55 Daxuecheng South Road, Chongqing, 401331 P.R. China \\ E-mail: ybshen@mail.ustc.edu.cn, zhangry@ustc.edu.cn, \\ mawg@ustc.edu.cn, lixz0818@mail.ustc.edu.cn, dayu@mail.ustc.edu.cn, \\ guoleicqu@cqu.edu.cn
}

ERRATUM TO: JHEP10(2015)186

ARXIV EPRINT: 1507.03693

Due to some errors in the calculation of the differential cross sections, we made the following corrections.

1. Figures 3-8 are replaced by the following corresponding revised figures, respectively.

2. The sixth sentence of the second paragraph of section 3.3 should be changed to "In the plotted $M_{W^{+} Z Z}$ region, the NLO QCD+EW relative corrections in the inclusive and exclusive event selection schemes range from $123 \%$ to $170 \%$ and from $-1 \%$ to $38 \%$, respectively.".

3. The third sentence of the third paragraph of section 3.3 should be changed to "From the figures we can see that the NLO QCD relative corrections in the inclusive and exclusive event collection schemes are about $165 \%$ and $33 \%$, respectively, at the position of $y_{W+Z Z}=0 . "$. 

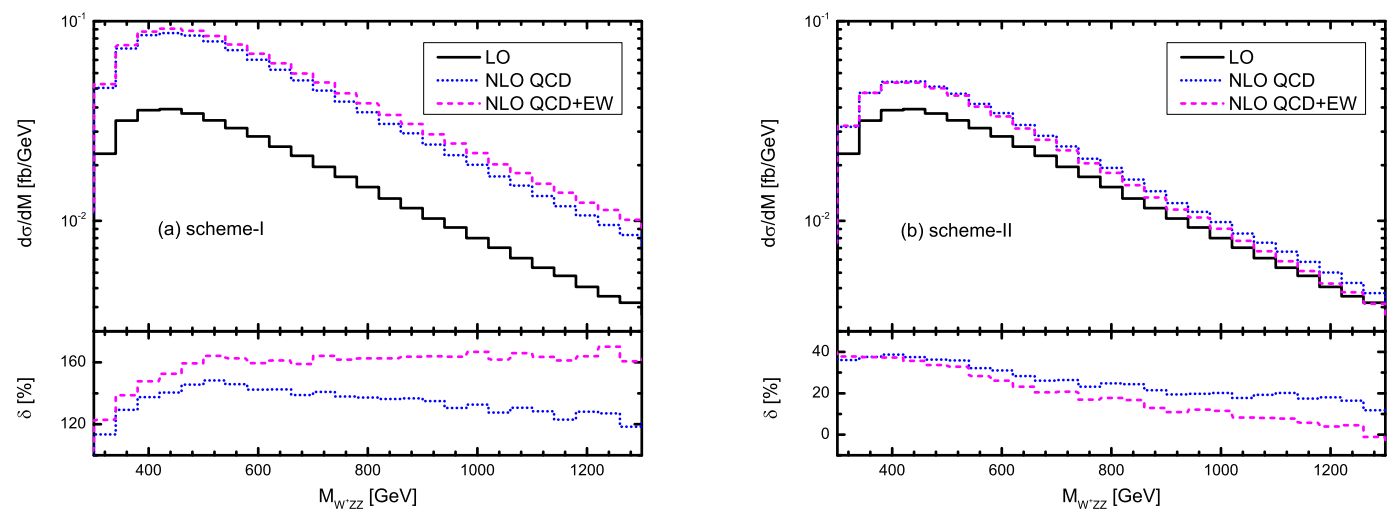

Figure 3. The $W^{+} Z Z$ invariant mass distributions $d \sigma_{\mathrm{LO}} / d M_{W^{+} Z Z}$ (solid), $d \sigma_{\mathrm{QCD}} / d M_{W^{+} Z Z}$ (dotted), $d \sigma_{\mathrm{NLO}} / d M_{W^{+} Z Z}$ (dashed) and the corresponding relative corrections for $p p \rightarrow W^{+} Z Z+X$ at the $\sqrt{S}=14 \mathrm{TeV}$ LHC in the (a) inclusive and (b) exclusive event selection schemes.
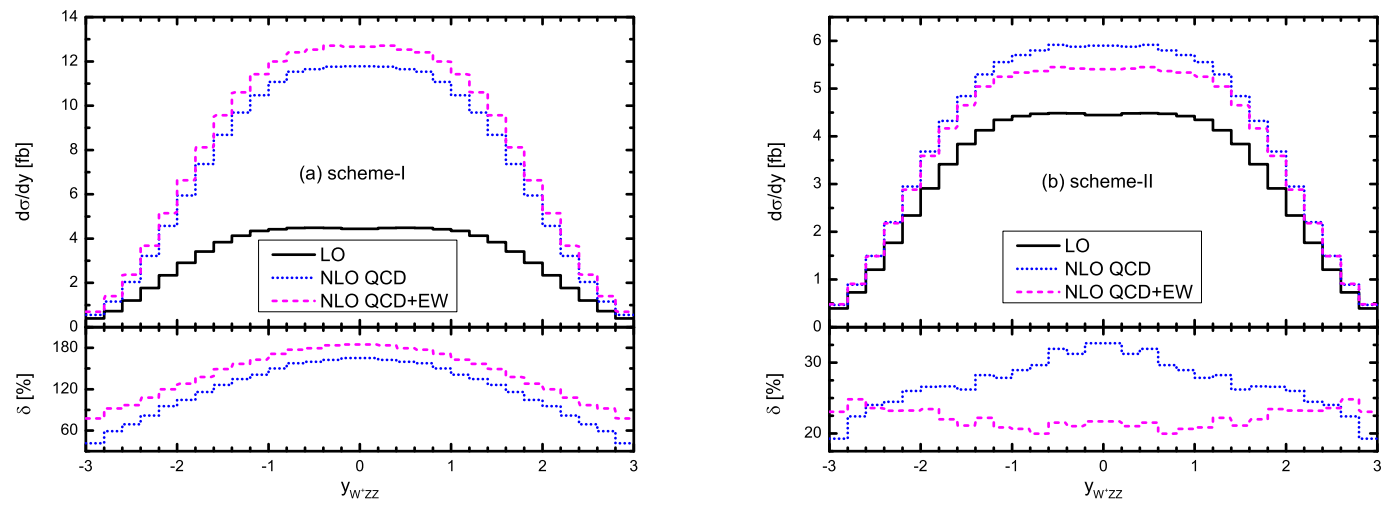

Figure 4. The $W^{+} Z Z$ rapidity distributions $d \sigma_{\mathrm{LO}} / d y_{W^{+} Z Z}$ (solid), $d \sigma_{\mathrm{QCD}} / d y_{W^{+} Z Z}$ (dotted), $d \sigma_{\mathrm{NLO}} / d y_{W+Z Z}$ (dashed) and the corresponding relative corrections for $p p \rightarrow W^{+} Z Z+X$ at the $\sqrt{S}=14 \mathrm{TeV}$ LHC in the (a) inclusive and (b) exclusive event selection schemes. 

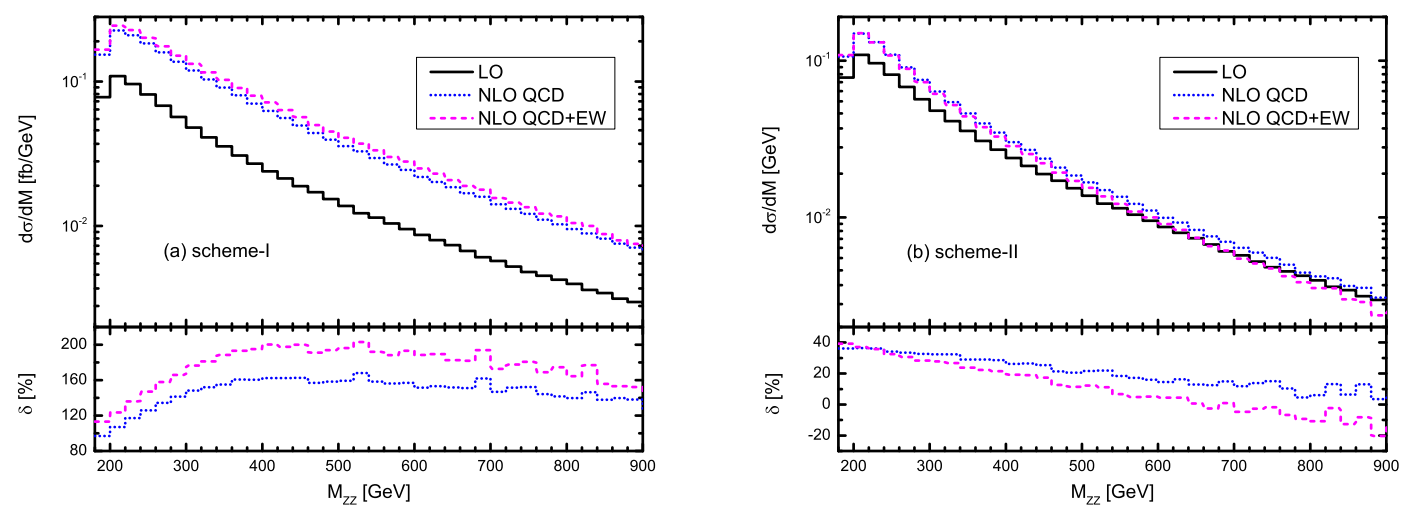

Figure 5. The $Z$-pair invariant mass distributions $d \sigma_{\mathrm{LO}} / d M_{Z Z}$ (solid), $d \sigma_{\mathrm{QCD}} / d M_{Z Z}$ (dotted), $d \sigma_{\mathrm{NLO}} / d M_{Z Z}$ (dashed) and the corresponding relative corrections for $p p \rightarrow W^{+} Z Z+X$ at the $\sqrt{S}=14 \mathrm{TeV}$ LHC in the (a) inclusive and (b) exclusive event selection schemes.
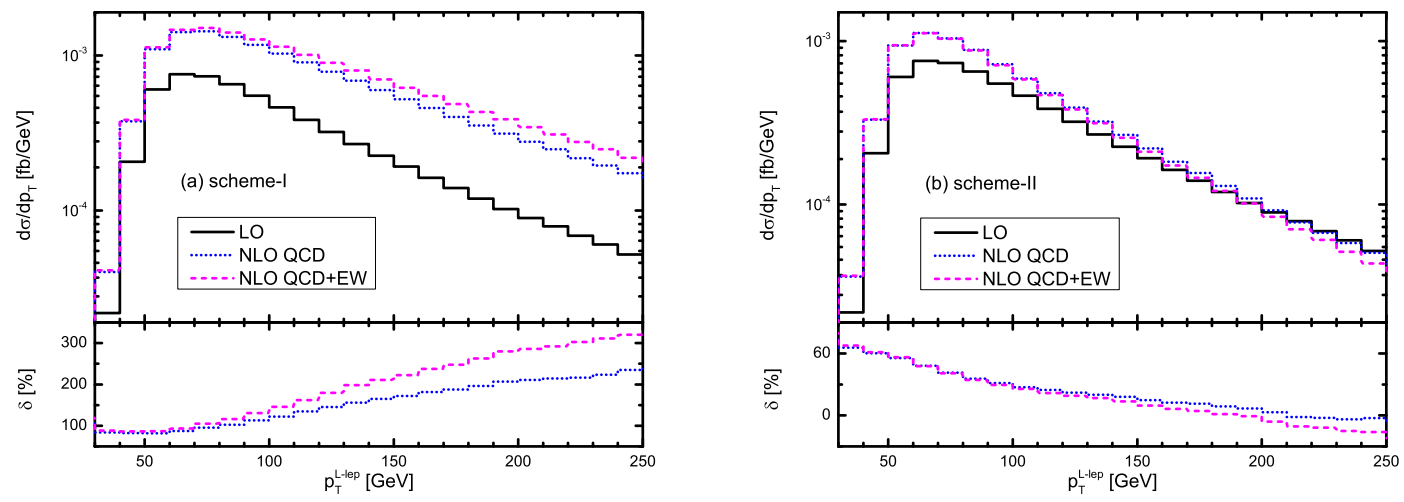

Figure 6. The leading lepton transverse momentum distributions $d \sigma_{\mathrm{LO}} / d p_{T}^{\mathrm{L}-l e p}$ (solid), $d \sigma_{\mathrm{QCD}} /$ $d p_{T}^{\text {L-lep }}$ (dotted), $d \sigma_{\mathrm{NLO}} / d p_{T}^{\mathrm{L}-l e p}$ (dashed) and the corresponding relative corrections for $p p \rightarrow$ $W^{+} Z Z \rightarrow \ell_{1}^{+} \nu_{\ell_{1}} \ell_{2}^{+} \ell_{2}^{-} \ell_{3}^{+} \ell_{3}^{-}+X$ at the $\sqrt{S}=14 \mathrm{TeV}$ LHC in the (a) inclusive and (b) exclusive event selection schemes. 

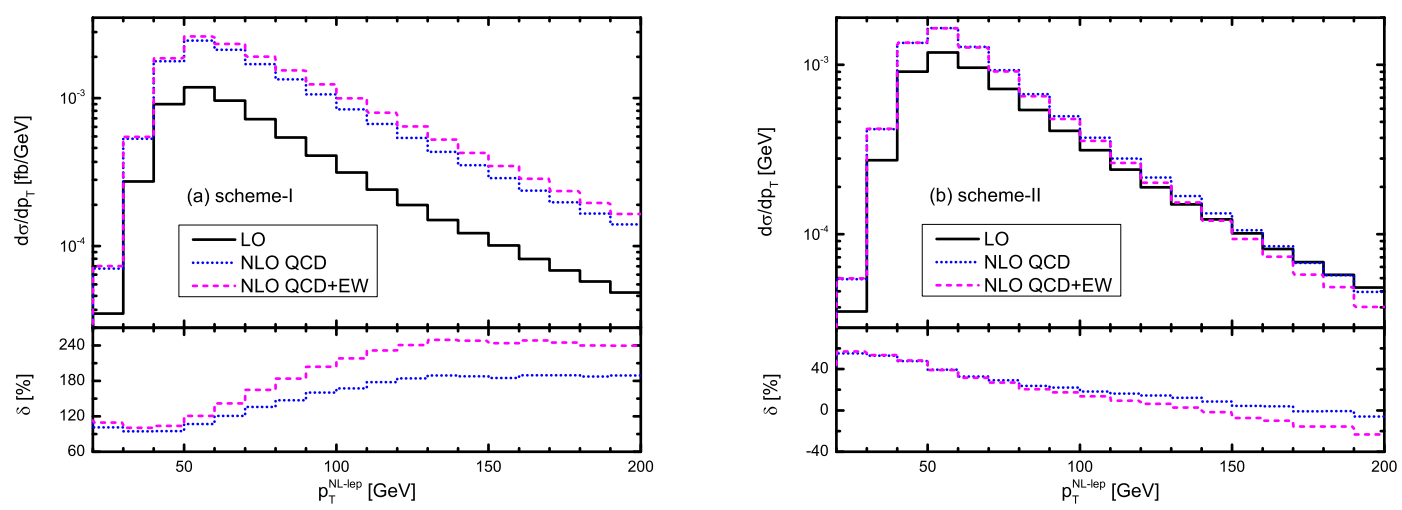

Figure 7. The next-to-leading lepton transverse momentum distributions $d \sigma_{\mathrm{LO}} / d p_{T}^{\text {NL-lep }}$ (solid), $d \sigma_{\mathrm{QCD}} / d p_{T}^{\mathrm{NL}-\mathrm{lep}}$ (dotted), $d \sigma_{\mathrm{NLO}} / d p_{T}^{\mathrm{NL}-\mathrm{lep}}$ (dashed) and the corresponding relative corrections for $p p \rightarrow W^{+} Z Z \rightarrow \ell_{1}^{+} \nu_{\ell_{1}} \ell_{2}^{+} \ell_{2}^{-} \ell_{3}^{+} \ell_{3}^{-}+X$ at the $\sqrt{S}=14 \mathrm{TeV}$ LHC in the (a) inclusive and (b) exclusive event selection schemes.
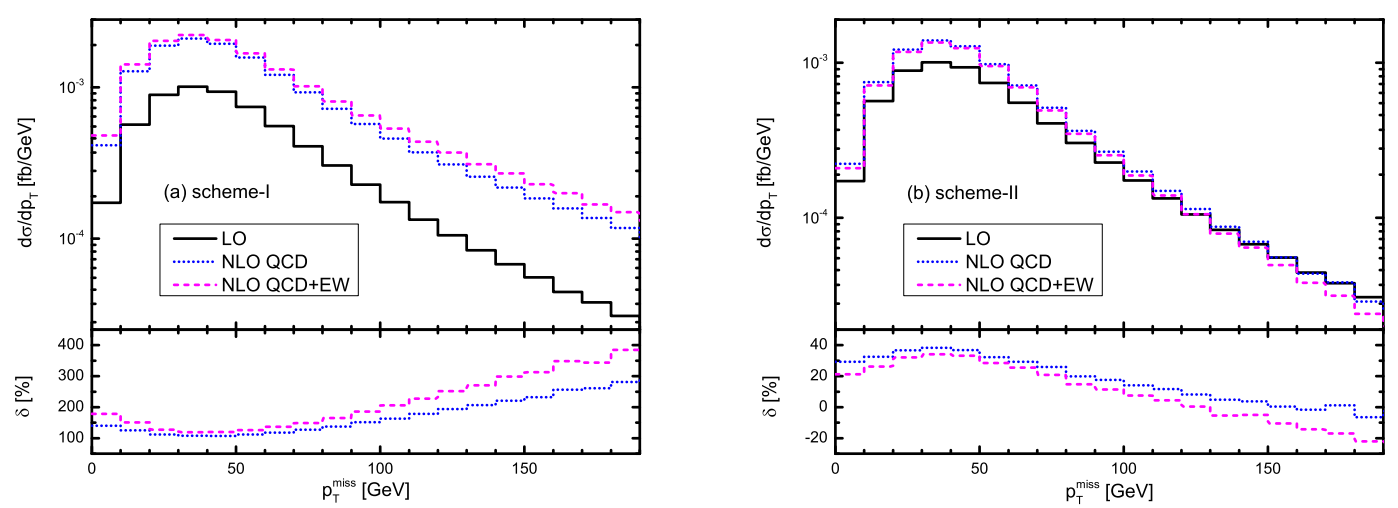

Figure 8. The missing transverse momentum distributions $d \sigma_{\mathrm{LO}} / d p_{T}^{\text {miss }}$ (solid), $d \sigma_{\mathrm{QCD}} / d p_{T}^{\text {miss }}$ (dotted), $d \sigma_{\mathrm{NLO}} / d p_{T}^{\text {miss }}$ (dashed) and the corresponding relative corrections for $p p \rightarrow W^{+} Z Z \rightarrow$ $\ell_{1}^{+} \nu_{\ell_{1}} \ell_{2}^{+} \ell_{2}^{-} \ell_{3}^{+} \ell_{3}^{-}+X$ at the $\sqrt{S}=14 \mathrm{TeV}$ LHC in the (a) inclusive and (b) exclusive event selection schemes. 
4. The fourth sentence of the fourth paragraph of section 3.3 should be changed to "The NLO QCD relative correction can exceed $138 \%$ when $M_{Z Z}>300 \mathrm{GeV}$ in the inclusive event collection scheme, and is less than $36 \%$ in the whole plotted $M_{Z Z}$ region in the exclusive event collection scheme.".

5. The fourth sentence from the bottom of the fifth paragraph of section 3.3 should be changed to "For example, we can read out from figure 6a that the NLO QCD relative correction in the inclusive event selection scheme increases from $82 \%$ to $235 \%$ as the increment of $p_{T}^{\mathrm{L}-\mathrm{lep}}$ from $50 \mathrm{GeV}$ to $250 \mathrm{GeV}$.".

Open Access. This article is distributed under the terms of the Creative Commons Attribution License (CC-BY 4.0), which permits any use, distribution and reproduction in any medium, provided the original author(s) and source are credited. 